\title{
Taking Advantage of Scattering Delocalization To Reduce Radiation Damage In Vibrational or Valence-Loss EELS and Energy-Filtered TEM Images
}

\author{
R.F. Egerton ${ }^{1}$, T. Aoki ${ }^{2}$ and P.A. Crozier ${ }^{3}$. \\ 1. Department of Physics, University of Alberta, Edmonton, Canada. \\ 2. LeRoy Eyring Center for Solid State Science, Arizona State University, Tempe, USA. \\ 3. School for the Engineering of Matter, Transport and Energy, Arizona State University, USA.
}

Delocalization of inelastic scattering, which limits the spatial resolution of EELS or EFTEM images, can be represented by a delocalization length $L_{50}$ or more fully by a point-spread function (PSF) that can be calculated from the angular distribution of the scattering [1]. Dipole scattering often predominates and its Lorentzian angular distribution (with characteristic angle $\theta_{\mathrm{E}}$ ) then gives rise to an approximately Lorentzian PSF with an exponential roll-off at a radial distance $r=b_{\max }=1 /\left(k_{0} \theta_{\mathrm{E}}\right), k_{0}$ being the incident wavenumber. Most of the scattering occurs between $r=b_{\max }$ and $r=b_{\min }=1 /\left(k_{0} \theta_{c}\right)$, where $\theta_{\mathrm{c}}$ is a cutoff angle: approximately $\left(2 \theta_{\mathrm{E}}\right)^{1 / 2}$ for electronic excitation. Between these limits, the PSF is proportional to $1 / r^{2}$, resulting in a median delocalization radius of $r_{50} \sim\left(b_{\min } b_{\max }\right)^{1 / 2}$.

The Table below shows these radii for an incident energy $E_{0}=60 \mathrm{keV}$ and various energy losses $E$. The inelastic scattering extends to hundreds of $\mathrm{nm}$ for vibrational losses $(E<0.5 \mathrm{eV})$ but only to $2-20 \mathrm{~nm}$ for valence-electron losses. Radiation damage in beam-sensitive materials (radiolysis) involves mainly valence-electron excitation, the mean energy loss in a light-element (e.g. organic) specimen being of the order of $40 \mathrm{eV}$. Consequently damage can be largely avoided by positioning an electron probe more than $20 \mathrm{~nm}$ beyond the edge of a specimen (aloof geometry), allowing vibrational-mode spectroscopy to be carried out on radiation-sensitive specimens in an aloof-beam mode [2,3].

\begin{tabular}{|c|c|c|c|c|}
\hline$E(\mathrm{eV})$ & $\theta_{\mathrm{E}} \sim E / 2 E_{0}(\mu \mathrm{rad})$ & $b_{\min }(\mathrm{nm})$ & $r_{50}(\mathrm{~nm})$ & $b_{\max }(\mathrm{nm})$ \\
\hline 0.1 & 0.88 & 0.6 & 23 & 880 \\
\hline 0.4 & 3.5 & 0.3 & 8 & 220 \\
\hline 6 & 53 & 0.075 & 1.0 & 14.6 \\
\hline 40 & 352 & 0.029 & 0.25 & 2.2 \\
\hline
\end{tabular}

In aloof spectroscopy, the EELS signal is delocalized over an area of approximately $2.5 b^{2}$, where $b$ is the beam-specimen separation [3], and energy-loss mapping within the specimen is not feasible. However it is still possible to take advantage of delocalization in the usual transmission mode, provided the energy loss of interest is less than the average value that gives rise to damage $(\sim 40 \mathrm{eV})$.

Green curves in Fig. 1 show the PSF for energy losses of $6 \mathrm{eV}$ ( $\pi^{*}$ excitation) and $0.45 \mathrm{eV}(\mathrm{O}-\mathrm{H}$ stretch). The blue curves are a PSF for damage, calculated by summing over all valence losses exceeding $5 \mathrm{eV}$, rather than taking an average loss of $40 \mathrm{eV}$. Red curves represent the PSF after damage, obtained by multiplying green curves by $\left.\exp \left[-D(r) / D_{\mathrm{c}}\right)\right]$, where $D(r)$ is the local accumulated dose (proportional to the blue curve) and $D_{\mathrm{c}}$ is a characteristic dose. Damage begins at small radii and spreads outwards. The energy-loss signal, obtained from integration of the red curve, decays quickly but then much more slowly as material outside a radius $\mathrm{r} \sim 2 \mathrm{~nm}$ damages at a much lower rate; see Fig. 2.

To take advantage of this behavior, a small $(<1 \mathrm{~nm})$ probe can be digitally rastered with a step size of 
several $\mathrm{nm}$. Energy-loss signal is then recorded from largely undamaged material between each probe position (see Fig. 2), providing a "chemical" image with a spatial resolution determined largely by delocalization. In this procedure, sub-pixel scanning (introduced to limit damage) must be turned off. [4] References:

[1] RF Egerton, EELS in the Electron Microscope, $3^{\text {rd }}$ Edition (Springer, 2011), p. 223.

[2] P Rez et al., submitted to Nature Communications (2016).

[3] RF Egerton, Ultramicroscopy 159 (2015), p.95.

[4] The authors acknowledge funding the Natural Sciences and Engineering Research Council of

Canada (NSERC) and the US Department of Energy (DE-SC0004954).
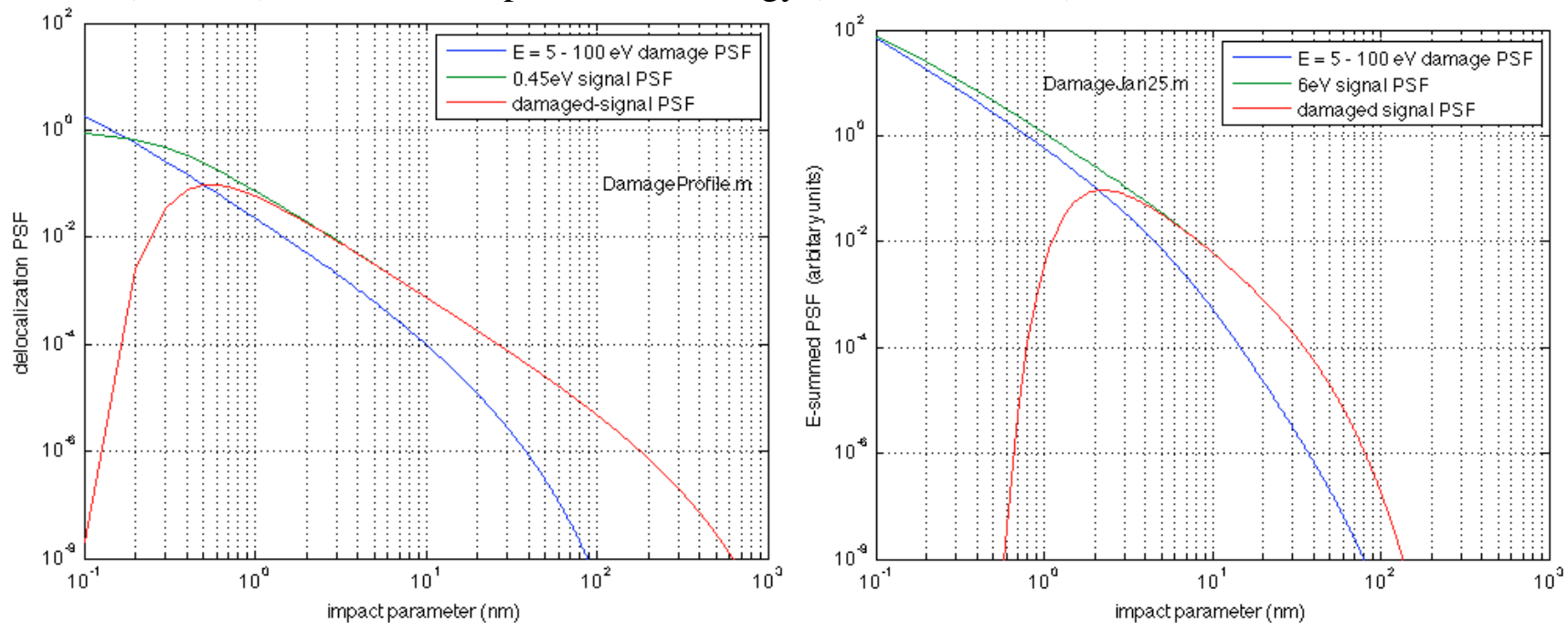

Figure 1. Point-spread function $\operatorname{PSF}(r, E)$ for damage (blue curve) and for EELS signals (green curve) at $0.45 \mathrm{eV}$ (left) and $6 \mathrm{eV}$ (right). Red curves show the $r$-dependence of signal after damage has occurred.
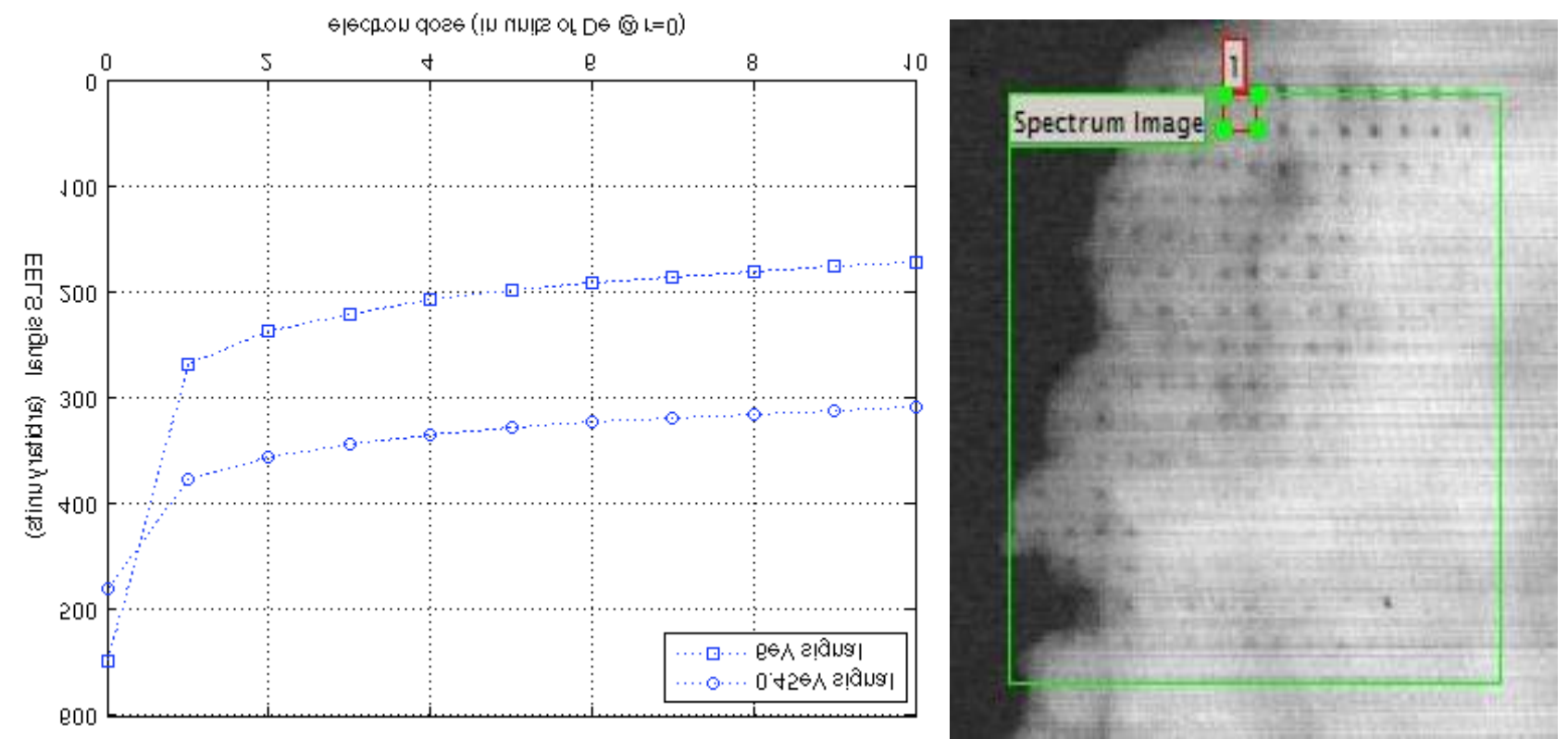

Figure 2. Left: $0.45 \mathrm{eV}$ and $6 \mathrm{eV}$ signals as a function of electron dose, from $r$-integration of Fig. 1. Right: HAADF image showing effect of a digital raster on a $\mathrm{Ca}(\mathrm{OH})_{2}$ test specimen. Size of the green square is $160 \mathrm{~nm} \times 160 \mathrm{~nm}$. Dark spots show positions of the electron beam, resulting in local mass-loss damage, whereas the vibrational-loss EELS signal remained largely unaltered after three complete scans. 